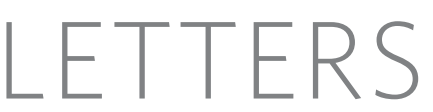

\title{
A flagellin-induced complex of the receptor FLS2 and BAK1 initiates plant defence
}

\author{
Delphine Chinchilla ${ }^{1}$, Cyril Zipfel ${ }^{1,2}$, Silke Robatzek ${ }^{1,3}$, Birgit Kemmerling $^{4}$, Thorsten Nürnberger ${ }^{4}$, \\ Jonathan D. G. Jones ${ }^{2}$, Georg Felix ${ }^{1,4} \&$ Thomas Boller $^{1}$
}

Plants sense potential microbial invaders by using patternrecognition receptors to recognize pathogen-associated molecular patterns (PAMPs) ${ }^{1}$. In Arabidopsis thaliana, the leucine-rich repeat receptor kinases flagellin-sensitive 2 (FLS2) (ref. 2) and elongation factor Tu receptor (EFR) (ref. 3) act as pattern-recognition receptors for the bacterial PAMPs flagellin ${ }^{4}$ and elongation factor $\mathrm{Tu}$ (EF-Tu) (ref. 5) and contribute to resistance against bacterial pathogens. Little is known about the molecular mechanisms that link receptor activation to intracellular signal transduction. Here we show that BAK1 (BRI1-associated receptor kinase 1), a leucinerich repeat receptor-like kinase that has been reported to regulate the brassinosteroid receptor BRI1 (refs 6,7), is involved in signalling by FLS2 and EFR. Plants carrying bak1 mutations show normal flagellin binding but abnormal early and late flagellintriggered responses, indicating that $\mathrm{BAK} 1$ acts as a positive regulator in signalling. The bak1-mutant plants also show a reduction in early, but not late, EF-Tu-triggered responses. The decrease in responses to PAMPs is not due to reduced sensitivity to brassinosteroids. We provide evidence that FLS2 and BAK1 form a complex in vivo, in a specific ligand-dependent manner, within the first minutes of stimulation with flagellin. Thus, BAK1 is not only associated with developmental regulation through the plant hormone receptor BRI1 (refs 6,7), but also has a functional role in PRR-dependent signalling, which initiates innate immunity.

PAMPs have key roles as activators of the innate immune response in animals ${ }^{8}$ and, analogously, as 'general elicitors' of defence responses in plants ${ }^{1-5,9-11}$. We have previously characterized FLS2 and EFR as the pattern-recognition receptors (PRRs) for flagellin (represented by a 22 -amino-acid peptide, flg22) and for EF-Tu (represented by the peptides elf18 and elf26, which correspond to its amino terminus), respectively ${ }^{2-5,10}$. Flagellin and EF-Tu rapidly induce a common set of Arabidopsis genes for leucine-rich repeat receptor-like kinases (LRR-RLKs), including FLS2 and EFR themselves $^{3,11}$. This led to the assumption that some of these PAMPinduced LRR-RLKs might encode additional components of PAMP perception or signalling. Using a reverse genetic approach, we tested a collection of insertional mutants in these LRR-RLKs (previously used for identification of the EFR gene ${ }^{3}$ ) for responsiveness to flg22 and found that two mutants with insertions in the LRR-RLK gene At4g33430 have reduced sensitivity to flg22 in seedling growth assays (Fig. 1a,b). In more than 10 repetitions of seedling growth assays with these mutants, we always observed a clear reduction (but never a complete loss) of sensitivity to flg22 and flg22-related peptides. By contrast, the mutants seemed to be as sensitive as the wild type to treatment with elf18 in more than five seedling growth assays (Fig. 1b and data not shown). a

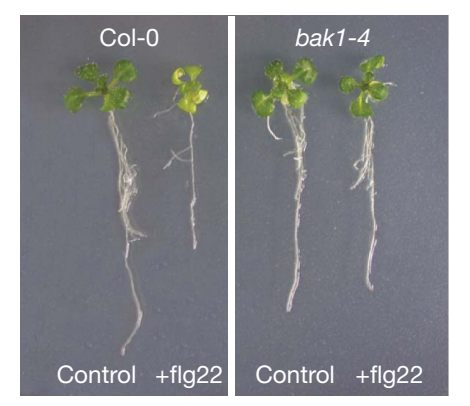

b

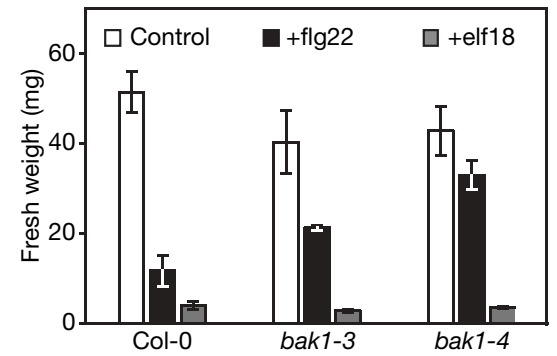

c

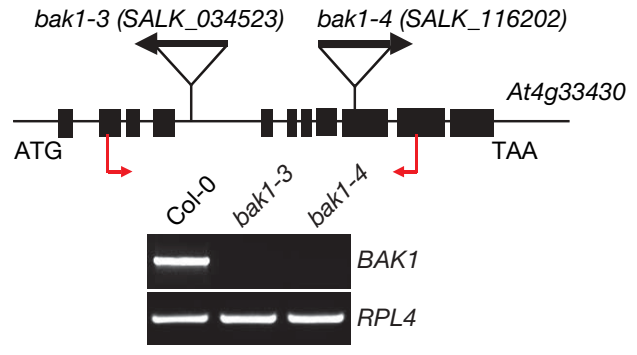

d
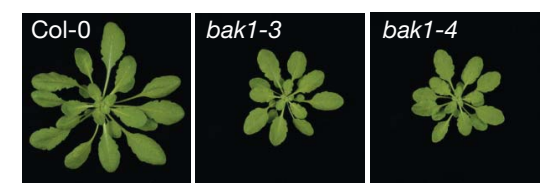

Figure 1 | bak1 mutants show reduced sensitivity to flagellin in growth assays. a, Wild-type (Col- 0$)$ and bak1-4 seedlings grown for 9 days in the presence of $10 \mathrm{nM} \mathrm{flg22.} \mathrm{b,} \mathrm{Seedling} \mathrm{growth} \mathrm{of} \mathrm{Col-0,} \mathrm{bak1-3} \mathrm{and} \mathrm{bak1-4} \mathrm{after}$ treatment with $10 \mathrm{nM}$ flg22 or $10 \mathrm{nM}$ elf18. Results shown are means \pm s.d. $(n=6)$.c, T-DNA insertion sites in bakl-3 and bakl-4 with exons shown as black boxes (top). RT-PCR analysis of BAK1 and RPL4 (control) transcripts in Col-0, bak1-3 and bak1-4 seedlings (bottom). Primers indicated by red arrows were used to test for the presence of a full-length $B A K 1$ transcript. d, Col-0, bak1-3 and bak1-4 plants, photographed 4 weeks after germination. 

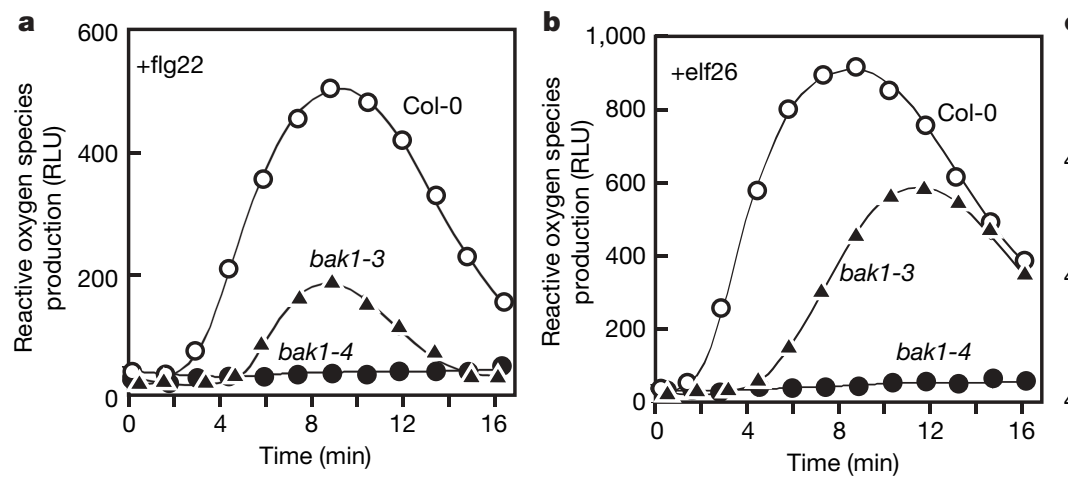

c

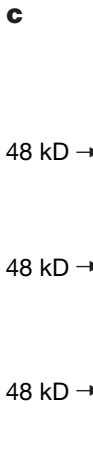

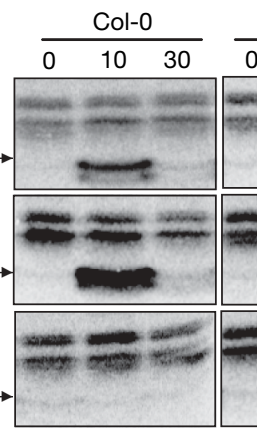

bak1-4

$\begin{array}{lll}0 & 10 & 30 \\ \end{array}$

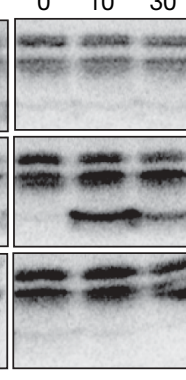

$+\mathrm{flg} 22$

+ elf18

Control
Figure 2 | bak1 mutants are impaired in responsiveness to flagellin and EF-Tu. a, Oxidative burst induced by $10 \mathrm{nM}$ flg22, measured in relative light units (RLU) in leaves of wild-type (Col-0), bak1-3 and bak1-4 plants. Results shown are means of six replicates. b, Oxidative burst induced by $10 \mathrm{nM}$ elf 26 . Results shown are means of six replicates. c, MBP kinase activity in Col-0

The LRR-RLK that is encoded by At4g33430 has been shown to interact with the brassinosteroid receptor BRI1 and was therefore named BRI1-associated receptor kinase 1 (BAK1) ${ }^{6,7}$. We consequently renamed the allelic lines SALK_034523 and SALK_116202 as bak1-3 and bak1-4, respectively (Fig. 1c). Both T-DNA insertion lines lacked the signal for BAK1 mRNA that is seen in wild-type plants when tested with appropriate primers by PCR with reverse transcription (RT-PCR; Fig. 1c) or by northern blot analysis (Supplementary Fig. 1). Complementation of bak1 mutants with wild-type $B A K 1$ restored their responsiveness to flg22 (data not shown).

Brassinolide and other brassinosteroids are structurally related to the animal steroid hormones and control many aspects of plant growth and development ${ }^{12}$. The two bak1 mutants that have been previously characterized show a semi-dwarf phenotype and reduced sensitivity to brassinolide ${ }^{6,7}$. Similarly, the bak1-3 and bak1-4 mutant lines showed reduced size when grown in soil (Fig. 1d). In our standard growth assays to measure long-term flg22 and elf18 responses $^{2,3}$, exogenous brassinolide applied at $10 \mathrm{nM}$ had a marginal growthpromoting effect in wild-type but not in bak1 mutant seedlings (Supplementary Fig. 2a). Treatments with $100 \mathrm{nM}$ and $1 \mu \mathrm{M}$ brassinolide stimulated the growth of bakl and wild-type seedlings to the same degree, showing that any bakl-mediated defect was overcome by brassinolide at $100 \mathrm{nM}$ or more. In the presence of $100 \mathrm{nM}$ brassinolide, flg22 still inhibited the growth of wild-type much more than bak1-4 seedlings (Supplementary Fig. 2b), indicating that the growth inhibition that is induced by flg22 is independent of brassinosteroid signalling. Corroborating this conclusion, cabbage 1 (cbb1) mutant plants, which are defective in brassinosteroid synthesis ${ }^{13}$, were as sensitive to flg22 as were wild-type plants (Supplementary Fig. 2c,d). plants and bak1-4 mutants after mock treatment (control) or treatment with $1 \mu \mathrm{M}$ flg22 ${ }^{\text {Xac }}$ or $1 \mu \mathrm{M}$ elf18. Gels were loaded with equal amounts of protein; no radiolabelled bands were observed in the parts of the gel omitted from the figure.

To test whether BAK1 has a direct role in flagellin signalling, we studied some of the early responses that are triggered by PAMPs in plants. Wild-type plants responded rapidly to flg22 with the induction of an oxidative burst (Fig. 2a). This response was clearly reduced and delayed in bak1-3 plants and almost abolished in bak1-4 mutants. Interestingly, the oxidative burst triggered by elf26 was also impaired in bak1-3 and bak1-4 mutants (Fig. 2b), providing evidence that mutations in bak1 also affect EF-Tu responses. Signalling in response to flagellin and EF-Tu involves the rapid activation of MAP kinases $(\mathrm{MAPK})^{3}$. In-gel assays with myelin basic protein (MBP) as a substrate showed that activation of MAPK was delayed and reduced or even absent after stimulation with EF-Tu or flagellin in the bak1-4 mutant, when compared to the wild type (Fig. 2c). These results show that signalling and early responses to flg22 and EF-Tu are affected in bak1 mutants. Therefore, we propose that BAK1 is a positive regulator of PAMP signalling in Arabidopsis.

The reduced sensitivity of bak1 mutants to flagellin might be due to a reduction in the expression or function of the flagellin receptor FLS2. However, western blotting detected similar amounts of FLS2 protein in wild-type and bak1 mutant plants (Fig. 3a). In addition, the amount and affinity of functional receptor binding sites were similar in wild-type and bak1 plants (Fig. 3b,c). Similarly, bak1 mutants have been reported to show normal binding of brassinolide to BRI1 (ref. 14). Thus, BAK1 seems to regulate the function of both receptors, BRI1 and FLS2, at a step after ligand binding. There is evidence that BAK1 is involved in BRI1 endocytosis ${ }^{15}$, and we recently found that FLS2 undergoes ligand-induced endocytosis ${ }^{16}$. Compared to wild-type plants, bak1-3 mutants showed markedly reduced endocytosis of FLS2 (Supplementary Fig. 3).
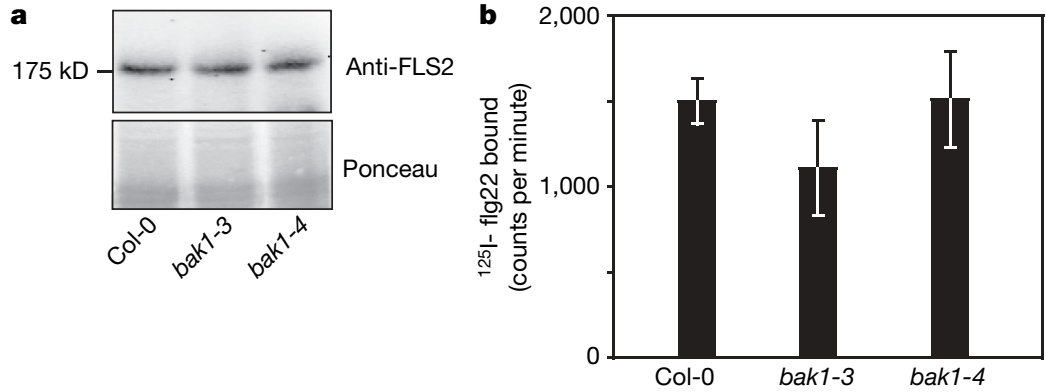

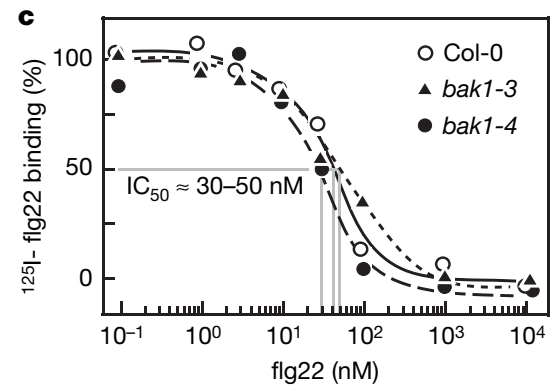

Figure 3 | Flg22 binding sites are unaffected in bak1 mutants. a, Western blot analysis of extracts from wild-type (Col-0), bak1-3 and bak1-4 seedlings with anti-FLS2 antibodies (top). Ponceau stain of the blot shows equal loading (bottom). b, Abundance of flagellin receptor binding sites in Col-0, bak1-3 and bak1-4 plants, estimated by specific binding of ${ }^{125} \mathrm{I}-\mathrm{flg} 22$. Results shown are means \pm s.d. $(n=3)$. c, Competitive binding assays in bak1-3, bak1-4 and Col-0. Binding of ${ }^{125} \mathrm{I}$-flg22 was tested in the presence of different concentrations of unlabelled flg22. The concentration of flg22 required to reduce binding by $50 \%\left(\mathrm{IC}_{50}\right)$ is indicated by the vertical grey lines. 
To test whether BAK1 interacts with FLS2, we used transgenic plants expressing Myc-tagged BAK1 in co-immunoprecipitation experiments (Fig. 4). Immunoprecipitates with anti-FLS2 antibodies revealed very little BAK1-Myc in untreated controls (Fig. 4). By contrast, the immunoprecipitates from plants stimulated with flg22 contained much more BAK1-Myc, indicating that FLS2 interacts with BAK1 in a stimulus-dependent manner (Fig. 4). BAK1-Myc could not be detected in immunoprecipitates with control antibodies (Fig. 4a), or in anti-FLS2 immunoprecipitates from non-transgenic plants (Fig. 4d). The reciprocal immunoprecipitation using anti-Myc antibodies confirmed the flg22-dependent heteromerization of BAK1 and FLS2 (Fig. 4b). We used immunoprecipitates such as those presented in Fig. 4a,b to study flg22 binding (Fig. 4c). Immunoprecipitates obtained with anti-FLS2 antibodies contained similar, large amounts of FLS2-binding sites, regardless of flg22 treatment. Thus, FLS2 binds flg22 independently of its association with BAK1. Immunoprecipitates obtained with anti-Myc antibodies prepared from untreated plants did not contain FLS2 (Fig. 4b) and had negligible flg22 binding (Fig. 4c), showing that BAK1 by itself does not bind flg22. By contrast, anti-Myc immunoprecipitates from flg22treated cells contained FLS2 (Fig. 4b) and were able to bind flg22 (Fig. 4c). Fewer binding sites were detectable in this precipitate than in the immunoprecipitates generated with anti-FLS2 antibodies, indicating that only a fraction of the FLS2 present in the solubilisate was pulled down in association with BAK1. Ligand-induced formation of the FLS2-BAK1 complex was specific for flg22 and did not occur after stimulation of the plants with elf26 (Fig. 4d) or treatment with brassinolide (Fig. 4e). Moreover, the antagonist peptide flg22$\Delta 2$, which binds FLS2 without activating a response ${ }^{10}$, failed to induce complex formation (Fig. 4e). Pre-treatment with the protein kinase inhibitor K-252a, which inhibits elicitor responses ${ }^{4,17}$, reduced the flg22-induced formation of the complex between the two serine/ threonine kinases ${ }^{6,718}$ BAK1 and FLS2 (Fig. 4f). We could not trigger an association of FLS2 and BAK1 in vitro by adding flg22 to extracts of untreated plants (data not shown). In vivo, however, complex formation occurred rapidly-within 2 min of treatment with flg22 (Fig. 4g). These kinetics are consistent with the idea that the FLS2-BAK1 complex is involved in receptor activation, as the earliest flg22-triggered responses can be measured after about $2 \mathrm{~min}$ of lag phase $^{4}$.

Ligand-induced dimerization is important for the activation of receptor kinases in animals ${ }^{19,20}$. Interestingly, classifying receptor kinases according to the occurrence of an arginine-aspartic acid (RD) motif in the catalytic site, FLS2 belongs to the non-RD class and BAK1 to the RD class ${ }^{21}$. This extends the similarity of signalling by PRRs in plants and animals highlighted in a recent review ${ }^{21}$ : PAMP recognition in animals through Toll-like receptors initially involves interaction with interleukin-1 receptor-associated kinase 1 (IRAK1), a non-RD kinase, and subsequently with IRAK4, an RD kinase.

BAK1 also associates with BRI1 (refs 22,23), an RD kinase ${ }^{21}$. However, the physiological responses that are triggered by BRI1 and FLS2 are very different. Therefore, BAK1 probably does not determine the specificity of the signal output; rather, it is likely to have a common role as an adaptor or co-receptor for the regulation of various receptors. Recent findings also show that bakl mutants have altered susceptibility to microbial pathogens such as oomycetes and true fungi ${ }^{24}$, indicating that BAK1 might regulate PRRs other than FLS2 and EFR.

Clearly, bak1 mutants retain partial sensitivity to flagellin and EF-Tu. One possible explanation for this is that closely related proteins might substitute for BAK1: BAK1 is also named SERK3 because it belongs to the SERK (somatic embryogenesis receptor-like kinase) family, which comprises five closely related LRR-RLKs ${ }^{25}$. The different SERKs might be partially functionally redundant, as reported for SERK1 and SERK2 (refs 26,27). BRI1 was recently found in complex with SERK1 (ref. 28), indicating that BAK1 might be substituted by other SERKs. In contrast to the serk3 (bak1) mutants described in this report, however, the other single mutants serk1, serk2, serk 4 and serk5 showed no defect in flg22 and elf18 responses (data not shown). In ongoing work we are trying to establish mutants in multiple SERK a
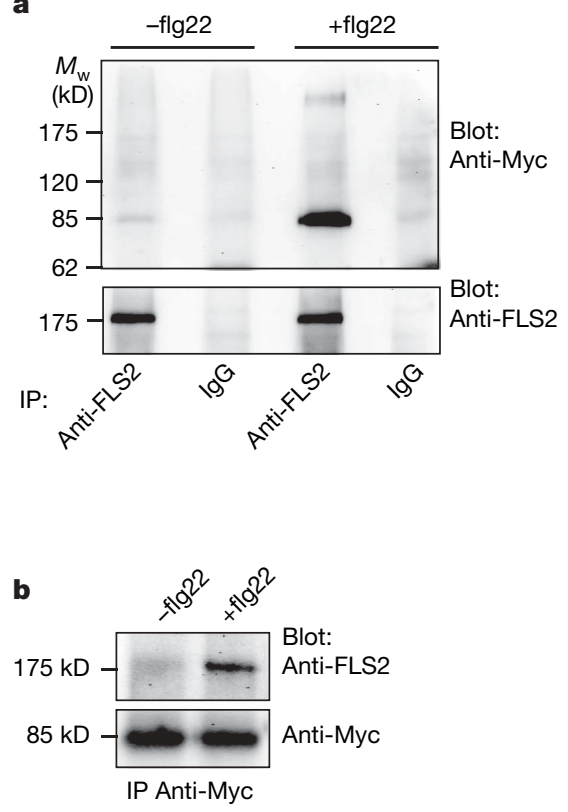

c

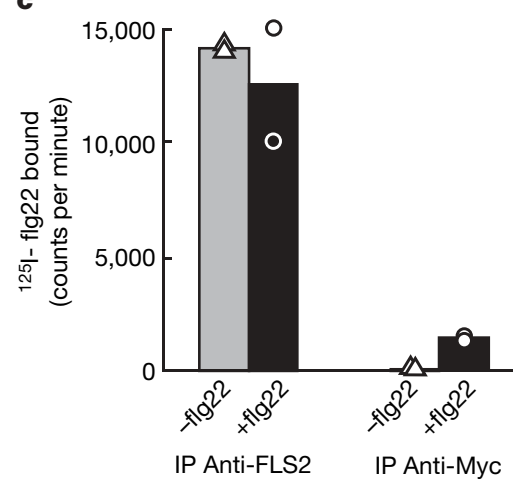

d

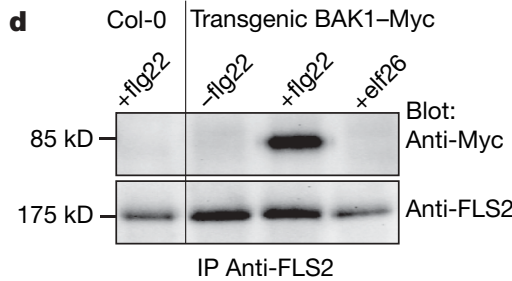

e
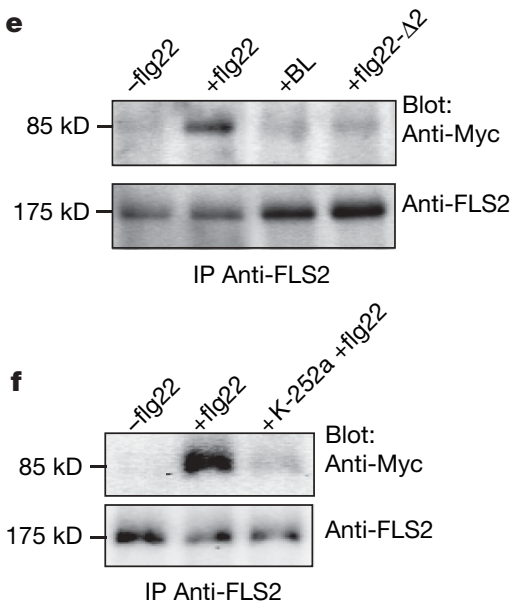

g

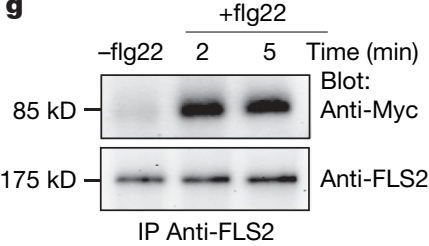

Figure 4 | FLS2 rapidly interacts with BAK1 in a ligand-dependent manner. a, Seedlings expressing BAK1-Myc were treated (or not) with flg22 for $5 \mathrm{~min}$. Solubilized membrane proteins were immunoprecipitated (IP) with antiFLS2 or control antibodies (IgG). Top: western blot with anti-Myc antibodies. Bottom: re-analysis of the blot with anti-FLS2 antibodies. b, Reciprocal co-immunoprecipitation with anti-Myc antibodies. c, Specific
${ }^{125} \mathrm{I}$-flg22 binding in anti-FLS2 and anti-Myc IPs from plants treated (or not) with $1 \mu \mathrm{M}$ flg22. Results are means of two samples. d, Formation of FLS2-BAK1 complex is specific for induction by flg22. e, Complex formation does not occur after treatment with the antagonist flg22- $\Delta 2$ or brassinolide (BL). f, Pre-treatment with the protein kinase inhibitor K-252a reduces complex formation. $\mathrm{g}$, Time course of complex formation. 
genes. However, as found for the serk 3 serk 4 double mutants, which die within 2 weeks of germination (data not shown, and J. Li, personal communication), this straightforward genetic approach might be impeded by essential functions carried out by the SERK proteins.

In conclusion, receptor activation of FLS2 by its ligand flagellin involves rapid complex formation with BAK1. Our results widen the scope for BAK1 function in a way reminiscent of the TOLL receptor in Drosophila, which controls embryo development as well as innate immunity ${ }^{29}$ : BAK1 has at least two roles in plants, being a positive regulator of PAMP receptors (thus influencing innate immunity) and of the plant hormone receptor BRI1 (thus influencing development).

\section{METHODS SUMMARY}

The Arabidopsis plants used in this study were grown as one plant per pot at $20-21{ }^{\circ} \mathrm{C}$ with an $8 \mathrm{~h}$ photoperiod, or on plates containing MS salts medium (Duchefa), $1 \%$ sucrose, and $0.8 \%$ agar under continuous light. The BAK1 T-DNA insertion lines SALK_034523 (bakl-3) and SALK_116202 (bak1-4) were generated by SIGnAL ${ }^{30}$ and obtained from the Nottingham Arabidopsis Stock Centre (Nottingham, UK). BAK1- and T-DNA-specific primers were used to select plants homozygous for the inserts. cbbl (cabbage 1) mutant seeds ${ }^{13}$ were obtained from the NASC collection. The flagellin peptide flg22, flg22- $\Delta 2$ and the EF-Tu peptides elf18 and elf26 used in this study have been described ${ }^{4,5,11}$. The peptide flg22 ${ }^{\text {Xac }}$ (QRLSSGLRINSAKDDAAGLAIS), which is equivalent in its action to flg22, was synthesized according to the sequence of the flg22-domain in Xanthomonas axonopodis pv. citri.

Assays for seedling growth inhibition, oxidative burst, in-gel MBP protein kinase activity, flg22-binding assays and western blot analysis with anti-FLS2 antibodies were performed as described ${ }^{4,5,10,11}$.

For co-immunoprecipitation experiments, seedlings transformed with a pBAK1-BAK1-Myc construct were extracted and immunoprecipitated with anti-FLS2 antibodies, anti-Myc polyclonal antibodies (Upstate), or anti-GFP rabbit serum (Molecular Probes). Immunoprecipitates were analysed by western blot with anti-Myc polyclonal antibodies or FLS2 antibodies.

Full Methods and any associated references are available in the online version of the paper at www.nature.com/nature.

Received 27 April; accepted 7 June 2007.

Published online 11 July 2007.

1. Nürnberger, T., Brunner, F., Kemmerling, B. \& Piater, L. Innate immunity in plants and animals: striking similarities and obvious differences. Immunol. Rev. 198, 249-266 (2004).

2. Gómez-Gómez, L. \& Boller, T. FLS2: an LRR receptor-like kinase involved in the perception of the bacterial elicitor flagellin in Arabidopsis. Mol. Cell 5, 1003-1011 (2000).

3. Zipfel, C. et al. Perception of the bacterial PAMP EF-Tu by the receptor EFR restricts agrobacterium-mediated transformation. Cell 125, 749-760 (2006).

4. Felix, G., Duran, J. D., Volko, S. \& Boller, T. Plants have a sensitive perception system for the most conserved domain of bacterial flagellin. Plant J. 18, 265-276 (1999).

5. Kunze, G. et al. The $\mathrm{N}$ terminus of bacterial elongation factor Tu elicits innate immunity in Arabidopsis plants. Plant Cell 16, 3496-3507 (2004).

6. $\mathrm{Li}$, J. et al. BAK1, an Arabidopsis LRR receptor-like protein kinase, interacts with BRI1 and modulates brassinosteroid signalling. Cell 110, 213-222 (2002).

7. Nam, K. H. \& Li, J. BRI1/BAK1, a receptor kinase pair mediating brassinosteroid signalling. Cell 110, 203-212 (2002).

8. Janeway, C. A. Jr \& Medzhitov, R. Innate immune recognition. Annu. Rev. Immunol. 20, 197-216 (2002)

9. Zeidler, D. et al. Innate immunity in Arabidopsis thaliana: lipopolysaccharides activate nitric oxide synthase (NOS) and induce defense genes. Proc. Natl Acad. Sci. USA 101, 15811-15816 (2004).
10. Chinchilla, D., Bauer, Z., Regenass, M., Boller, T. \& Felix, G. The Arabidopsis receptor kinase $\mathrm{FLS} 2$ binds $\mathrm{flg} 22$ and determines the specificity of flagellin perception. Plant Cell 18, 465-476 (2006).

11. Zipfel, C. et al. Bacterial disease resistance in Arabidopsis through flagellin perception. Nature 428, 764-767 (2004).

12. Vert, G., Nemhauser, J. L., Geldner, N., Hong, F. \& Chory, J. Molecular mechanisms of steroid hormone signalling in plants. Annu. Rev. Cell Dev. Biol. 21, 177-201 (2005).

13. Kauschmann, A. et al. Genetic evidence for an essential role of brassinosteroids in plant development. Plant J. 9, 701-713 (1996).

14. Kinoshita, T. et al. Binding of brassinosteroids to the extracellular domain of plant receptor kinase BRI1. Nature 433, 167-171 (2005).

15. Russinova, E. et al. Heterodimerization and endocytosis of Arabidopsis brassinosteroid receptors BRI1 and AtSERK3 (BAK1). Plant Cell 16, 3216-3229 (2004).

16. Robatzek, S., Chinchilla, D. \& Boller, T. Ligand-induced endocytosis of the pattern recognition receptor FLS2 in Arabidopsis. Genes Dev. 20, 537-542 (2006).

17. Felix, G., Grosskopf, D. G., Regenass, M. \& Boller, T. Rapid changes of protein phosphorylation are involved in transduction of the elicitor signal in plant cells. Proc. Natl Acad. Sci. USA 88, 8831-8834 (1991).

18. Gómez-Gómez, L., Bauer, Z. \& Boller, T. Both the extracellular leucine-rich repeat domain and the kinase activity of FSL2 are required for flagellin binding and signalling in Arabidopsis. Plant Cell 13, 1155-1163 (2001).

19. Massague, J. TGF-beta signal transduction. Annu. Rev. Biochem. 67, 753-791 (1998).

20. Schlessinger, J. Ligand-induced, receptor-mediated dimerization and activation of EGF receptor. Cell 110, 669-672 (2002).

21. Dardick, C. \& Ronald, P. Plant and animal pathogen recognition receptors signal through non-RD kinases. PLoS Pathogens 2, e2 (2006).

22. Li, J. \& Jin, H. Regulation of brassinosteroid signalling. Trends Plant Sci. 12, 37-41 (2007).

23. Wang, X. et al. Identification and functional analysis of in vivo phosphorylation sites of the Arabidopsis BRASSINOSTEROID-INSENSITIVE1 receptor kinase. Plant Cell 17, 1685-1703 (2005).

24. Kemmerling, B. et al. The BRI1-associated kinase 1, BAK1, has a brassinolideindependent role in plant cell-death control. Curr. Biol. doi:10.1016/ j.cub.2007.05.046 (published online 21 June 2007).

25. Hecht, V. et al. The Arabidopsis SOMATIC EMBRYOGENESIS RECEPTOR KINASE 1 gene is expressed in developing ovules and embryos and enhances embryogenic competence in culture. Plant Physiol. 127, 803-816 (2001).

26. Colcombet, J., Boisson-Dernier, A., Ros-Palau, R., Vera, C. E. \& Schroeder, J. I. Arabidopsis SOMATIC EMBRYOGENESIS RECEPTOR KINASES1 and 2 are essential for tapetum development and microspore maturation. Plant Cell 17, 3350-3361 (2005).

27. Albrecht, C., Russinova, E., Hecht, V., Baaijens, E. \& de Vries, S. The Arabidopsis thaliana SOMATIC EMBRYOGENESIS RECEPTOR-LIKE KINASES1 and 2 control male sporogenesis. Plant Cell 17, 3337-3349 (2005).

28. Karlova, R. et al. The Arabidopsis SOMATIC EMBRYOGENESIS RECEPTOR-LIKE KINASE1 protein complex includes BRASSINO STEROID-INSENSITIVE1. Plant Cell 18, 626-638 (2006).

29. Lemaitre, B., Nicolas, E., Michaut, L., Reichhart, J. M. \& Hoffmann, J. A. The dorsoventral regulatory gene cassette spatzle/Toll/cactus controls the potent antifungal response in Drosophila adults. Cell 86, 973-983 (1996).

30. Alonso, J. M. et al. Genome-wide insertional mutagenesis of Arabidopsis thaliana. Science 301, 653-657 (2003).

Supplementary Information is linked to the online version of the paper at www.nature.com/nature.

Acknowledgements We thank $A$. Bent and V. Lipka for critically reading the manuscript; S. Salomon and A. Caniard for technical help; and the Salk Institute Genomic Analysis Laboratory (SIGnAL) and the NASC stock center for the T-DNA insertion lines and $c b b 1$ seeds. This work was supported by the Swiss National Foundation and the European Molecular Biology Organization.

Author Information Reprints and permissions information is available at www.nature.com/reprints. The authors declare no competing financial interests. Correspondence and requests for materials should be addressed to D.C. (delphine.chinchilla@unibas.ch)or T.B. (thomas.boller@unibas.ch). 


\section{METHODS}

Plant material and growth conditions. Arabidopsis plants used in this study were grown as one plant per pot at $20-21{ }^{\circ} \mathrm{C}$ with an 8 -h photoperiod, or on plates containing MS salts medium (Duchefa), $1 \%$ sucrose, and $0.8 \%$ agar under continuous light. The BAK1 T-DNA insertion lines SALK_034523 (bak1-3) and SALK_116202 (bak1-4) were generated by SIGnAL ${ }^{30}$ and obtained from the Nottingham Arabidopsis Stock Centre (Nottingham, UK). BAK1- and T-DNAspecific primers were used to select plants homozygous for the inserts. $c b b 1$ (cabbage 1) mutant seeds ${ }^{13}$ were obtained from the NASC collection.

Materials. The flagellin peptide flg22, flg22- $\Delta 2$ and the EF-Tu peptides elf18 and elf26 used in this study have been described ${ }^{4,5,11}$. The peptide flg $22^{X a c}$ (QRLSSGLRINSAKDD AAGLAIS), which is equivalent in its action to flg22, was synthesized according to the sequence of the flg22-domain in Xanthomonas axonopodis pv. citri. Brassinolide (Sigma) and K-252a (Alexis) were prepared as stock solutions of $10 \mathrm{mM}$ in formamide and $2 \mathrm{mM}$ in DMSO, respectively.

Bioassays. Assays for seedling growth inhibition, oxidative burst and in-gel MBP protein kinase activity were performed as described ${ }^{4,5,11}$.

RT-PCR analysis. One microgram of DNase-treated RNA was reversetranscribed using Superscript II reverse transcriptase (Invitrogen), and one microlitre of the reaction mix was used for PCR amplification with 30 cycles and the $B A K 1$ primers that span the T-DNA insertion locations (5'-GGTGCTTCAAAGTTGGGATG-3' and 5'-GGCTTCAAACTCTT CATCCAACAAA$3^{\prime}$ ) or for the constitutively expressed control gene RPL4 (At1g07320 (5' TGATAGGTCAGGTCAGGGAACAAC-3' and 5' -CCACCACCACGAA CTTCACCGCG AGTC-3').

Binding assays. Binding assays with plant extracts were done as described ${ }^{10}$. Briefly, $100 \mathrm{mg}$ of leaves ground in liquid nitrogen were resuspended in $500 \mu \mathrm{l}$ of binding buffer (25 mM MES/KOH pH6.0, $3 \mathrm{mM} \mathrm{MgCl}_{2}, 10 \mathrm{mM} \mathrm{NaCl}$ ). Aliquots of $80 \mu \mathrm{l}$ of extract were incubated in binding buffer in a total volume of $100 \mu \mathrm{l}$ with ${ }^{125} \mathrm{I}$-flg22 ( $60 \mathrm{fmol}$ per standard assay, $>2,000 \mathrm{Ci} \mathrm{mmol}^{-1}$ ) for $25 \mathrm{~min}$ either alone (total binding) or with an excess of $10 \mu \mathrm{M}$ unlabelled flg22 (non-specific binding). After incubation for $25 \mathrm{~min}$ at $4{ }^{\circ} \mathrm{C}$, unbound ligand was removed by filtration and radioactivity retained on the filters was determined by $\gamma$-counting. To determine the specific binding, non-specific binding was subtracted from total binding.

Generation of transgenic plants. A $1-\mathrm{kb}$ fragment of the $B A K 1$ promoter was amplified from genomic DNA (wild-type Col-0) and introduced into the XbaI and BamHI sites of binary vector pGREENII/ T-0229 (ref. 31). The BAK1 gene was amplified by PCR from genomic DNA and cloned into the BamHI and XhoI sites of pGREENII; the 3xMyc tag was PCR amplified and cloned in XhoI of this construct. The final construct $B A K 1 p-B A K 1-3 x M y c$ was verified by sequencing, electroporated into Agrobacterium EHA101 containing the helper plasmid pSOUP ${ }^{31}$ and used to transform bak1-4 mutant plants. Plants of the T2 generation were chosen for the co-immunoprecipitation experiments.

Immunoprecipitation experiments. Seedlings ( $1 \mathrm{~g}$ fresh weight), grown for 2 weeks in liquid MS medium, were frozen in liquid nitrogen, extracted by grinding with mortar and pestle, and taken up in $2.5 \mathrm{ml}$ of cold extraction buffer ( $50 \mathrm{mM}$ Tris- $\mathrm{HCl} \mathrm{pH} 8,150 \mathrm{mM} \mathrm{NaCl}, 10 \%$ glycerol, 1\% (w/v) Nonidet P-40, $0.5 \%(\mathrm{w} / \mathrm{v})$ sodium deoxycholate and protease inhibitor cocktail (Sigma)). After incubation for $2 \mathrm{~h}$ at $4{ }^{\circ} \mathrm{C}$ with gentle shaking, this preparation was filtered through Miracloth and centrifuged at 40,000 r.p.m. for $30 \mathrm{~min}$. The supernatant was incubated overnight at $4{ }^{\circ} \mathrm{C}$ with proteinA-sepharose beads (Amersham Biosciences) and polyclonal anti-FLS2 antibodies, anti-Myc (Upstate), or antiGFP rabbit serum (Molecular Probes) used as control. The beads were collected and washed three times with ice-cold extraction buffer and once with $50 \mathrm{mM}$ Tris- $\mathrm{HCl} \mathrm{pH} \mathrm{7.5.} \mathrm{Proteins} \mathrm{that} \mathrm{were} \mathrm{retained} \mathrm{on} \mathrm{the} \mathrm{beads} \mathrm{were} \mathrm{separated} \mathrm{by}$ SDS-PAGE 7\% (w/v) and analysed by western blot with antibodies against Myc or FLS2 as described ${ }^{10}$. Unless indicated otherwise, seedlings were treated for 5 min with $10 \mu \mathrm{M}$ of peptides (flg 22 or elf 26 as indicated), with $1 \mu \mathrm{M}$ of flg $22-\Delta 2$, with $1 \mu \mathrm{M}$ of brassinolide or $1 \mu \mathrm{M}$ of $\mathrm{K}-252 \mathrm{a}$. Controls were run in parallel with application of the solvents alone (DMF for brassinolide and DMSO for K-252a, respectively).

31. Hellens, R. P., Edwards, E. A., Leyland, N. R., Bean, S. \& Mullineaux, P. M. pGreen: a versatile and flexible binary Ti vector for Agrobacterium-mediated plant transformation. Plant Mol. Biol. 42, 819-832 (2000). 